\title{
A theoretical analysis of altruism and decision error in public goods games
}

\author{
Simon P. Anderson, Jacob K. Goeree, Charles A. Holt* \\ Department of Economics, 114 Rouss Hall, University of Virginia, Charlottesville, VA 22903-3288, \\ USA
}

Received 30 June 1997; accepted 28 February 1998

\begin{abstract}
We formalize an equilibrium model in which altruism and decision-error parameters determine the distribution of contributions for linear and quadratic public goods games. The equilibrium density is exponential for linear games, and normal for quadratic games. Our model implies: (i) contributions increase with the marginal value of the public good, (ii) total contributions increase with the number of participants, (iii) mean contributions lie between the Nash prediction and half the endowment. These predictions, which are not implied by a Nash analysis, are consistent with laboratory data. Maximum likelihood estimates of altruism and error parameters are significant and plausible. (C) 1998 Elsevier Science S.A. All rights reserved.
\end{abstract}

Keywords: Altruism; Decision error; Experiments; Public goods games; Logit equilibrium JEL classification: C92; $\mathrm{H} 41$

\section{Introduction}

A public goods game with a linear payoff structure produces extreme Nash equilibria - participants should contribute nothing if the marginal value of the public good is less than the cost of contributing, and as much as possible otherwise. The game is particularly simple in that these are dominant strategies. However, laboratory experiments with this game suggest that subjects tend to

\footnotetext{
*Corresponding author. Fax: +1 8049822904.
} 
follow less extreme strategies, contributing more than predicted when the equilibrium is at zero contributions, and less than predicted when the equilibrium is at full contributions. Moreover, the observed level of contributions is typically increasing in the marginal private value of the public good and in the number of players, although (non-critical) changes in these parameters do not affect the Nash equilibrium. Although average contributions typically decline over time, full 'free riding' is usually not observed even after as many as a 60 rounds. ${ }^{1}$

The presence of significant levels of voluntary contributions when full free riding is a dominant strategy is sometimes attributed to altruism. However, if the Nash equilibrium is at the zero-contribution boundary, then any 'noise' or decision error will also bias contributions upward. Moreover, just adding altruism cannot explain why subjects do not contribute their entire endowments when full contribution is the Nash equilibrium. Although a number of papers have evaluated these and other explanations of behavior, there seems to be no consensus about the relative importance of noise, altruism, and other factors.

Much of the debate has concentrated on altruism, and there is conflicting evidence as to the form of altruism felt by subjects. The major dichotomy is between 'pure altruism' (individuals who care about others' payoffs) and 'warmglow' altruism (individuals enjoy contributing per se). Andreoni (1993) notes that public contributions would crowd out private contributions if pure altruism were predominant. However, since such crowding out was far from complete in both his experiments and the field data he cites, he concludes that warm-glow effects are significant. Likewise, Offerman et al. (1996a) report evidence of warm-glow effects in step-level public goods games.

However, a number of other papers present evidence for pure altruism in laboratory experiments. Andreoni and Miller (1995) report that a significant proportion of subjects make altruistic decisions when choosing between combinations of money payments for themselves and other participants. Moreover, the extent of the transfers to others depends on the terms of the tradeoff between own and others' earnings. With only warm-glow altruism, the amount of money transferred to others would not depend on these terms of trade. Even more persuasive is the fact that contributions in public goods experiments rise with increases in the group size, at least for low marginal values of the public good (Isaac and Walker, 1988; Isaac et al., 1994). When people only care about the act of giving and not about how much good it does, such numbers effects are not present. Of course, it is possible that there is some mix of warm-glow and pure altruism effects. Sefton and Steinberg (1996) conclude from their public goods experiment with a quadratic structure that there is evidence of altruism, yet Laury (1997) reaches the opposite conclusion based on Laury et al. (1997) experiments with a quadratic structure. Below we consider both linear and quadratic structures

\footnotetext{
${ }^{1}$ For surveys of this literature, see Ledyard (1995) and Davis and Holt, 1993 (chapter 6). Holt and Laury (1997) review alternative theoretical explanations of these deviations from Nash predictions.
} 
and attempt to resolve some of these different points of view, using theoretical and empirical methods.

A couple of recent papers explicitly attempt to disentangle altruism and error effects. Andreoni (1996) changed the basic voluntary contributions game by paying each subject according to the rank of the subject's earnings, rather than according to the dollar amount of earnings. This manipulation reduces the levels of contributions dramatically. Since the rank-based payments add a constant-sum element to the game, this treatment removes incentives for reciprocal altruism and collusion. The large reduction in contributions suggests the importance of pure altruism or 'kindness' in the standard payoff format (which is not rank-based). The relatively low levels of contributions that remain in the rank-payoff treatment are ascribed to 'confusion.' Andreoni's paper suggests that both altruism and errors are present in the standard game, but because the whole structure of the game has been changed, it is not clear that the residual deviation from Nash equilibrium in the rank-based game is a measure of errors in the standard public goods game. One objective of this paper is to analyze a formal equilibrium model for the standard contributions game that simultaneously allows the possibility of pure altruism, warm-glow, and decision error. The model provides a way to evaluate the relative importance of these factors.

Palfrey and Prisbrey (1997) provide an alternative way to disentangle the effects of errors and the types of altruism. These authors estimate a probit model for contributions in a binary choice public goods game. They conclude that there is a fair amount of noise in the decision process, and that warm-glow effects are significant (albeit low in magnitude), but find no evidence of pure altruism. We compare our empirical results to theirs below.

The theoretical treatment of pure altruism here is a straightforward incorporation of others' payoffs into the utility functions, an approach that follows Edgeworth (1881); DeGroot and Cyert (1973) more recently, Ledyard (1995); Palfrey and Prisbrey (1997). Warm-glow is incorporated via a direct utility benefit from contribution. The treatment of decision errors is based on the standard logit model in which choice probabilities are determined by expected payoffs, so that more costly errors are less likely to occur. ${ }^{2}$ The model is parameterized so as to allow perfect rationality as a limit case. Since players make mistakes, each player's expected payoff depends on the distribution of others' actions. The equilibrium distribution of actions is consistent in the sense that it is a fixed point: the distribution of actions determines expected payoffs, which in turn determine the distribution of actions via the standard logit formula. Hence, the resulting logit equilibrium distribution is endogenously determined by the structural payoff parameters of the game.

\footnotetext{
${ }^{2}$ Mirrlees (1986) proposes using the logit form to analyze boundedly rational behavior (although not in a game setting). Anderson et al. (1992) survey the origins of the logit model and its economic applications.
} 
This is an example of what McKelvey and Palfrey (1995), in a path-breaking paper, have termed a 'quantal response equilibrium'. 'They analyze the properties of quantal response equilibria for finite games, proving existence for a general formulation and then specializing the discussion to a logit form to establish limit properties (in particular, a Nash equilibrium is attained at the limit when decisions tend to perfect rationality, but not all Nash equilibria can be attained in this manner). McKelvey and Palfrey (1995); Ochs (1995) use the logit model to explain the results of several experiments based on finite games. Similarly, Offerman et al. (1996b) use the logit model in their analysis of a step-level public good game in which subjects make binary contribution decisions and the public good is provided only if enough subjects contribute. In a like vein, Palfrey and Prisbrey (1997) use a binary probit analysis: for the binomial case the probit is very similar to the logit. By contrast, our approach is based on a continuous formulation of the logit model. The continuous version was previously used by Lopez (1995) to analyze auction games.

The logit equilibrium model is not explicitly dynamic, and therefore, it does not explain features of laboratory data that arise because of interactions across periods, for example the fact that contributions tend to fall over time. Two proposed explanations for this latter tendency are termed 'simple learning' and 'strategies' by Andreoni (1988). The first explanation - subjects learn over time that lower contributions are (unilaterally) more profitable and so gravitate to lower levels - is rejected because there is a strong 'restart' effect in both Andreoni's (1988) data and the replication by Croson (1996). The strategies hypothesis is based on the 'crazy-player' justification for trigger strategies in a finitely repeated game, first formulated by Kreps et al. (1982). In the original prisoners' dilemma formulation (which is a special case of the contribution game, with two strategies), there is a

\footnotetext{
${ }^{3}$ Rosenthal (1989) provides an earlier theoretical analysis of boundedly rational equilibria using a linear form for the choice probabilities. Chen et al. (1997) introduce decision error in a learning model using a probabilistic choice function due to Luce (1959). There are different motivations for the use of probabilistic choice models in the literature. McKelvey and Palfrey (1995) mainly interpret the noise as unobserved shocks to utility, so that (in line with a standard interpretation of econometric discrete choice models) individuals maximize their (true) utilities, even though behavior may be inconsistent with payoffs as observed by an outsider. In contrast, Rosenthal (1989); Chen et al. (1997) seem to prefer an interpretation of latent or subconscious utility whereby individuals do not (or cannot) explicitly evaluate the utilities associated with the various options, but instead have a vague idea of what is best. The approaches are mathematically equivalent, so we do not need to espouse one or the other here.

${ }^{4}$ Expected payoffs in the step-level public good game depend on beliefs about others' contributions (in contrast to the linear public goods game analyzed here). Offerman et al. (1996a) compare the logit equilibrium model with a learning model in which there is naive Bayesian updating and logistic decision error. The learning model yields a better fit than the logit equilibrium model, which is not surprising since there is considerable adjustment during initial periods of non-equilibrium behavior. In the next section, we discuss an adjustment process that has the logit equilibrium as a steady state.

${ }^{5}$ In a restart treatment, subjects are told at the end of the formal session that there is time for another session that is then played out.
} 
positive probability that a player will cooperate as long as the other has in the past. It is then an equilibrium for rational players to mimic this behavior in early rounds of play, although they ultimately will not cooperate, and use mixed strategies towards the end of the game. This means that players establish reputations for cooperation early on, and use trigger strategies in the sense of reverting to noncooperative play for the duration of the game in response to any deviation from cooperation. Because of the mixed strategies, a cross-section analysis would show average contributions falling over time in a partners treatment, but in a strangers treatment one would expect contributions only from 'crazy players' (since rational players have no incentives to build reputations).

Andreoni's data did not support this hypothesis because strangers contributed more than partners, although the replication by Croson (1996) gave the opposite result: strangers contributed significantly less than partners, supporting the strategies hypothesis. Both these authors found strong restart effects for both partners and strangers treatments. In the prisoners' dilemma version, one would not expect a restart effect (since only 'crazy players' would have contributed in the last round), and by extension one would not expect a restart effect for the contributions game either. However, the observed jump up at the restart point does support a broader (but more vague) notion of reciprocity whereby individuals respond in kind ('do unto others as you would have others do unto you').

One such theory is provided by Isaac et al. (1994) and Laury (1997) who posit that individuals may use forward-looking strategies in which they signal the desire to cooperate by contributing, and will continue with this behavior as long as expected payoffs exceed those that would be received if everyone free rides. Here the number of remaining rounds is important because a high contribution has a greater potential benefit when there are more rounds in which others can reciprocate. Indeed, Isaac et al. (1994) report data patterns that are more sensitive to the number of rounds remaining than to the number of rounds completed. This forward-looking theory is similar to the notion of 'cooperative gain-seeking' put forth by Brandts and Schram (1996): some players cooperate as long as their payoffs are higher than would be the case if all players would free ride.

Hence, in small groups tacit collusion may be established through 'signals', rewards, and punishments. These dynamic effects should be largely absent in experiments with random matching and little chance of 'contagion' (e.g., being rematched with some of the same people or with their previous partners). In this sense, our model (which does not have forward-looking components) would probably predict best in such environments. Since most experiments involve partners treatments (fixed groupings), we acknowledge in advance that our approach will not be able to explain data patterns due to strategic behavior. However, one data pattern that is commonly observed in laboratory experiments,

\footnotetext{
${ }^{6}$ Kelley and Stahelski (1970) argue that players who are basically cooperative will adapt their behavior to the type of rival they face.
} 
i.e. the decline in contributions over time, can be explained by our approach. In the next section we discuss a 'noisy' adjustment model for which the steady state is the logit equilibrium. This dynamic model that underlies the logit equilibrium provides a plausible explanation for why contributions (which are close to 50 percent of the endowment in the first period) decrease over time.

The analysis of the equilibrium distribution of contributions in a linear public goods game is presented in Section 2, both with and without altruism. We show that contributions are stochastically increasing in the marginal value of the public good (a feature observed in many laboratory experiments) even though the Nash equilibrium is unaffected by (non-critical) changes in this parameter. In the presence of (pure) altruism, contributions are stochastically increasing in the number of players, again in contrast with the Nash prediction but consistent with the data. Hence both pure altruism and errors are needed to explain observed patterns. We use data from experiments by Isaac and Walker (1988); Isaac et al. (1994) to estimate error and pure altruism parameters. In contrast to Palfrey and Prisbrey (1997), the data display a significant amount of noise and pure altruism, but provide no evidence for the presence of warm-glow altruism.

In Section 3 we extend the discussion to quadratic public goods games. Even though there is a continuum of Nash equilibria in this model, there is a unique, symmetric logit equilibrium, which is a truncated normal distribution. Furthermore, mean contributions are 'sandwiched' between the Nash equilibrium and the midpoint of the range of feasible choices. This pattern is consistent with data from previous experiments. The final section summarizes and qualifies the conclusions.

\section{The linear public goods game}

The simple public goods game involves $n$ risk-neutral players, each of whom has an endowment of $\omega$ dollars. Players decide simultaneously how much of the endowment to contribute to the public good. If player $i$ contributes $x_{i}$ dollars, each player (including $i$ ) receives a payoff of $m x_{i}$ dollars from that contribution. Thus the payoff to player $i, \pi_{i}$, depends on all contributions: $\pi_{i}=\omega-x_{i}+m X$, where $m>0$ and $X$ is the sum of all contributions, including that of player $i$. The residual $\omega-x_{i}$ can be thought of as consumption of a private good. When $m<1$, the unique Nash equilibrium entails each player making a zero contribution, which is a dominant strategy. This game has a similar structure to a prisoner's dilemma for $1 / n<m<1$, since total payoffs are maximized by each player contributing $\omega$. When $m=1$, there is a continuum of Nash equilibria with each player contributing any amount of the endowment. When $m>1$, the unique Nash equilibrium coincides with the joint-payoff maximum at full contribution, which is again a strictly dominant strategy. 


\subsection{The equilibrium error model}

If indeed utility is given by the payoff formula above $\left(\pi_{i}=\omega-x_{i}+m X\right)$, any positive contribution is an error when $m<1$, and the severity of the error is greater the smaller is $m$. Likewise, contributing less than the endowment, $\omega$, is an error when $m>1$. We model errors in a way such that the magnitude of the expected contribution error is sensitive to the economic consequences. For example, if $m$ is small, the public good is relatively worthless and the model predicts that the contribution will be small.

Decision errors could be due to confusion about the payoff structure and/or errors in calculation. A different interpretation is that individuals' utilities contain random components that are unobservable to an outsider. This is the standard econometric view of fully rational behavior that does not maximize observed payoffs. Regardless of the source of 'error', we posit that relatively costly mistakes are less likely. The precise model we use for the error structure is a continuous version of the logit form, so that player $i$ 's probability density of choosing decision $x_{i}$ is a function of the expected profit $\pi_{i}^{\mathrm{e}}\left(x_{i}\right):^{7}$

$$
f_{i}\left(x_{i}\right)=\frac{\exp \left(\pi_{i}^{\mathrm{e}}\left(x_{i}\right) / \mu\right)}{\int_{0}^{\omega} \exp \left(\pi_{i}^{e}(x) / \mu\right) \mathrm{d} x}, \quad u=1, \ldots, n,
$$

where $\mu>0$ parameterizes the propensity to make errors. ${ }^{8}$ The integral in Eq. (1) is independent of $x_{i}$ and ensures that the density integrates to one. From this formula, actions with higher expected payoffs are more likely to be chosen. As the error parameter $\mu$ tends to zero, the probability of choosing the decision with the highest payoff tends to one, and so the decision converges to the rational one. The larger is $\mu$, the greater the degree of irrationality: if $\mu$ tends to infinity, the density

\footnotetext{
${ }^{7}$ The continuity of the contributions decision is for technical convenience, although the laboratory experiments are conducted with discrete choices. The endowments in the experiments that we discuss typically range from 10 to 62 . We believe that such endowments are large enough to make continuity a reasonable assumption, but whether our model would provide better predictions for experiments with continuous choices is an empirical question.

${ }^{8}$ This footnote presents a simple derivation of the logit model when there are $D$ decisions, with expected payoffs $U_{1}, \ldots, U_{D}$. Probabilistic discrete choice theory postulates that a person chooses decision $i$ if $U_{i}+\epsilon_{i}>U_{k}+\epsilon_{k}$, for all $k \neq i$, where the $\epsilon$ are idiosyncratic errors. These errors allow the possibility that the decision with the highest payoff will not be selected, and the probability of such an error depends on both the magnitude of the difference in the expected payoffs and on the 'spread' in the error distribution. The logit model results from assuming an i.i.d. double exponential distribution for the errors. Under this assumption, the probability of choosing decision $i$ is: $\exp \left(U_{i} / \mu\right) / \Sigma_{j=1, \ldots D}$ $\exp \left(U_{j} / \mu\right)$, where $\mu>0$ is proportional to the standard deviation of the error distribution. Alternatively, the logit model can be derived from basic choice axioms (Anderson et al., 1992).
} 
function in Eq. (1) becomes flat over its support and behavior becomes random (the probability density converges to $1 / \omega$, regardless of payoffs).

The equilibrium density can be readily calculated from Eq. (1) and the expected profit

$$
\pi_{i}^{\mathrm{e}}\left(x_{i}\right)=\omega-x_{i}+m x_{i}+m \sum_{j \neq i} E_{i}\left(x_{j}\right), \quad i=1, \ldots n,
$$

where $E_{i}\left(x_{j}\right)$ denotes the contribution that player $i$ expects from player $j$. Since $\omega$ and the expected contributions from other players enter additively in Eq. (2), they can be cancelled out of the exponential expressions of both the numerator and denominator of Eq. (1). This eliminates all player-specific terms, and the equilibrium density of the contribution $x$ is the same for all players:

$$
f(x)=K \exp (-(1-m) x / \mu)=K \exp (-\lambda x)
$$

where $\lambda=(1-m) / \mu$, and the constant $K$ ensures that the density integrates to one. Equating the integral of Eq. (3) over $[0, \omega]$ to one, we can determine $K$ and express Eq. (3) as:

$$
f(x)=\frac{\lambda \exp (-\lambda x)}{1-\exp (-\lambda \omega)}, \quad x \in[0, \omega]
$$

which is a truncated exponential density on $[0, \omega] .{ }^{9}$ The parameter $\lambda$ is proportional to the marginal cost of contribution, $1-m$. If $m<1$ (the benefit from contribution is less than its cost), the Nash equilibrium, which involves no errors, is to contribute nothing. However, the model with errors entails a probability density that does not have all its mass at zero, but declines away from zero. Hence there is a smaller probability of making a more severe error. For $m>1$ (so $\lambda=(1-m) / \mu$ is negative), the error density rises towards the Nash equilibrium outcome of full contribution. It is only in the perfect-rationality limit as $\mu$ converges to zero that the extreme outcomes of the Nash equilibrium are attained.

One of the most interesting qualitative predictions of the model pertains to the size of the mean contribution. If $\lambda>0$, the exponential density in Eq. (4) is decreasing on $[0, \omega]$, so the mean contribution lies between 0 (the Nash equilibrium) and $\omega / 2$. Conversely, for negative $\lambda$, the density is increasing on $[0$, $\omega$, so the mean contribution is between $\omega / 2$ and $\omega$ (the Nash equilibrium for $\lambda<0$ ). In both cases, the mean contribution lies between the Nash equilibrium and half the endowment. As we show in Section 3, this 'sandwich' property extends to the quadratic public goods game.

The density Eq. (4) can be integrated to obtain the associated cumulative distribution:

${ }^{9}$ Eq. (4) holds for $\lambda \neq 0$. If $\lambda=0(m=1)$, then we can determine directly from Eq. (3) that $K=1 / \omega$, and the equilibrium density is uniform over its support. 


$$
F(x)=\frac{1-\exp (-\lambda x)}{1-\exp (-\lambda \omega)}, x \in[0, \omega] .
$$

An analysis of this distribution yields the following proposition.

Proposition 1. The logit equilibrium for the linear public goods game (in the absence of altruism) has the following properties:

1. An increase in the marginal value of the public good leads to a stochastic increase in contributions;

2. An increase in the error rate leads to a stochastic increase in contributions when $m<1$, and a stochastic decrease in contributions when $m>1$;

3. An increase in the number of players has no effect on individual contributions;

4. An increase in the endowment leads to a stochastic increase in contributions.

Proof. The first two statements follow from the property that $F(x)$ in Eq. (5) is increasing in $X$ for all interior $x$, which is proved in Appendix A (Lemma 1). Hence the distribution for a given value of $\lambda$ dominates those for lower values, in the sense of first-degree stochastic dominance. Since $\lambda=(1-m) / \mu$, an increase in $m$ reduces $\lambda$ and thus shifts the cumulative distribution down, resulting in a stochastic increase of contributions. If $m<1$, an increase in the error rate $\mu$ reduces $\lambda$, which also shifts the distribution function down. This effect is reversed when $m>1$. Statement (iii) follows since both $\lambda$ and $F$ are independent of the number of players, $n$, whereas (iv) follows since the right side of Eq. (5) decreases with $\omega$. Note that first-degree stochastic dominance implies a lower mean contribution since the mean is the integral of $1-F(x)$. Q.E.D.

One feature of the data that is not covered by Proposition 1 is the tendency for contributions to decline over time, most markedly for low values of $\mathrm{m}$. Several explanations have been proposed for this observed decline. For instance, people might become more rational over time, or less altruistic when they observe that others free ride. Miller and Andreoni (1991) use a standard replicator model of evolutionary behavior to explain the decline in contributions; free riders become more prevalent over time because their payoffs exceed the average payoff. We also offer an evolutionary explanation, which is more closely related to the logit equilibrium approach. Anderson et al. (1997) specify a model in which players adjust their decisions in the direction of increasing payoff, but these adjustments are subject to error. More precisely, the time derivative of a person's contribution is assumed to be equal to the derivative of expected payoff plus a normal random shock (Brownian motion). We prove that this process converges over time to the logit equilibrium, independent of the initial distributions of decisions. In most laboratory experiments the initial contributions are quite dispersed, with the mean contribution close to fifty percent of the endowment. As contributions evolve in the direction of higher payoff, the average contribution falls to the logit 
equilibrium level which lies between the Nash level (zero contribution) and half the endowment. This observation also explains why contributions may actually rise when the mean of the logit equilibrium is relatively high (i.e. higher than the mean of the initial distribution of contributions).

To summarize the main findings of this section, an increase in the marginal value of the public good raises contributions in a model with decision errors, even though a (non-critical) increase has no effect on the Nash equilibrium. An increase in the propensity to err, $\mu$, evens out the probability density, and thus raises contributions when the Nash equilibrium is zero and lowers contributions when the Nash equilibrium is the full endowment. The endowment result in part (iv) of Proposition 1 is of interest because other evidence suggests that behavior in experiments depends on endowments even when the Nash equilibrium is unaffected. One example is the common resource pool problem described in Davis and Holt (1993). Closer to the present context, some experiments by Laury et al. (1997) showed that an increase in the individual endowment increased contributions to the public good (although their game form was the quadratic payoff structure treated in Section 3 below).

\subsection{Introducing altruism}

One property of the model above (without altruism) is that contributions are independent of the number of players in the game (Proposition 1(iii)). Olson (1971) suggests that there will be more free riding (lower contributions) with large groups in standard public goods environments. The experimental evidence on group size is limited, but Isaac and Walker (1988); Isaac et al. (1994) report higher individual contributions for larger groups in a linear public goods game. Pure altruism is one factor that may cause contributions to be higher in large groups: someone who cares about others may contribute more if there are more people to enjoy the public good.

We now extend the framework above to allow for altruism. To model pure altruism, we introduce a parameter $\alpha>0$ that represents an individual's utility weight on the payoff of other players. ${ }^{10}$ Warm-glow is modeled by adding a utility payoff term $g$ times the amount contributed. The utility of individual $i$ is now given by:

$$
U_{i}=\pi_{i}+g x_{i}+\alpha \sum_{j \neq i}^{n} \pi_{j} .
$$

Since $\pi_{i}=\omega-x_{i}+m X$, the term multiplying $x_{i}$ in Eq. (6) is $(g-1+m+m[n-$ $1] \alpha$ ), where $g-1$ reflects the marginal private cost of the contribution, $m$ is the

\footnotetext{
${ }^{10}$ This formulation was first used by Edgeworth (1881), who referred to $\alpha$ as the 'coefficient of sympathy.'
} 
marginal value of the public good, and $m[n-1] \alpha$ is the marginal value to all the other players, weighted by the pure altruism parameter. Since the expected payoff is linear in $x_{i}$, the (altruism-inclusive) Nash equilibrium is at zero if and only if $(g-1+m+m[n-1] \alpha)<0$. For $\alpha>0$ (altruism), this condition is satisfied if the number of players, $n$, is less than the critical level $n_{\mathrm{c}} \equiv 1+(1-m-g) /(\alpha m)$. Conversely, the Nash equilibrium involves full contribution, $\omega$, if the number of players exceeds this critical value.

This all-or-nothing prediction seems too stark, and is softened by introducing decision error. Indeed, for $\mu>0$, there is a positive probability that any strategy is played. As before, all terms independent of $x_{i}$ can be factored out of the numerator and denominator of Eq. (1). This yields a truncated exponential density as in Eq. (4), with $\lambda$ replaced by $\lambda^{*} \equiv(1-g-m-m[n-1] \alpha) / \mu$. The corresponding distribution is given by Eq. (5), also with $\lambda$ replaced by $\lambda^{*}$. The sign of $\lambda^{*}$ determines whether the density is decreasing or increasing in $x$, i.e. whether the mean contribution is relatively low or high. It is only in the limit when $\mu$ tends to zero that the density accumulates all its mass at the Nash solution $(0$ or $\omega)$.

In the presence of warm-glow alone $(g>0$ and $\alpha=0)$, an increase in the number of players has no effect on individual contributions because giving is valued per se $\left(\lambda^{*}\right.$ is unchanged with changes in $\left.n\right)$. However, with pure altruism $(\alpha>0)$, an increase in the number of players lowers $\lambda^{*}$ and the cumulative distribution, and therefore raises contributions in the sense of first-degree stochastic dominance. This implies that the more players there are, the greater are contributions, because the benefits from the public good are enjoyed by more people. Moreover, for any fixed number of players, the more people like giving, and the more intensely they care about others, the more they contribute. It follows from the structure of $\lambda^{*}$ and Eq. (5) that changes in $m$ and $\omega$ have the same qualitative effects as in the game without altruism. To summarize:

Proposition 2. In the logit equilibrium for the linear public goods game with pure altruism, an increase in the number of players leads to a stochastic increase in individual contributions, while there is no effect with only warm-glow altruism. The presence of pure and/or warm-glow altruism does not alter the qualitative comparative statics effects of increases in the endowment or in the marginal value of the public good; both lead to stochastic increases in contributions.

The results of Propositions 1 and 2, as compared with the stylized facts from some laboratory experiments, are presented on the left side of Table 1 . The first two rows show that three treatment variables $m, n, \omega$ are predicted to have no effect on the contributions in a Nash equilibrium. All three variables have a positive effect in a logit equilibrium with pure altruism, which is consistent with the available data (bottom row).

Although the model of decision errors could be enriched in a number of ways, the table suggests that the logit equilibrium provides qualitative predictions that 
Table 1

Summary comparative static results for the level of contributions

\begin{tabular}{|c|c|c|c|c|c|}
\hline \multirow[t]{2}{*}{ Parameters } & \multicolumn{3}{|c|}{$\begin{array}{l}\text { Linear game } \\
\text { (individual contributions) }\end{array}$} & \multicolumn{2}{|c|}{$\begin{array}{l}\text { Quadratic game } \\
\text { (total contributions) }\end{array}$} \\
\hline & $m$ & $n$ & $\omega$ & $n$ & $\omega$ \\
\hline Nash, $\alpha=0$ & 0 & 0 & 0 & 0 & 0 \\
\hline Nash, $\alpha>0$ & 0 & 0 & 0 & + & 0 \\
\hline Logit, $\alpha=0$ & + & 0 & + & $+{ }^{a}$ & + \\
\hline Logit, $\alpha>0$ & + & + & + & $+{ }^{\mathrm{b}}$ & + \\
\hline Data & $+{ }^{\mathrm{c}}$ & $+{ }^{d}$ & NA & $+{ }^{\mathrm{e}}$ & $+^{\mathrm{e}}$ \\
\hline
\end{tabular}

${ }^{\mathrm{a}}$ Total contributions rise even though individual contributions decline (see Proposition 5).

${ }^{\mathrm{b}}$ This is true for small $\alpha$ and/or small $\mu$, by continuity with the two preceding cases. We have not been able to show the result holds generally, though we strongly suspect this to be the case.

${ }^{\mathrm{c}}$ From surveys by Ledyard (1995); Davis and Holt (1993).

${ }^{\mathrm{d}}$ From Isaac and Walker (1988); Isaac et al. (1994).

${ }^{\text {e }}$ From Laury et al. (1997).

are superior to those of a Nash equilibrium in this context. This claim can be further substantiated econometrically. The closed-form solution for the equilibrium density in Eq. (4), with $\lambda$ replaced by the altruism inclusive form $\lambda^{*} \equiv(1-g-m-$ $m[n-1] \alpha) / \mu$, can be used to obtain maximum likelihood estimates of altruism and error parameters. We have analyzed the laboratory data from experiments conducted by Isaac and Walker (1988); Isaac et al. (1994). ${ }^{11}$ In a two-parameter model the estimated value of $\alpha$ is 0.067 (0.001) and that of $\mu$ is $0.026(0.001)$, where the numbers in parentheses are the standard errors. Hence there is significant evidence for the presence of pure altruism and errors. However, adding warm-glow altruism to the model resulted in a negative and insignificant estimate for $g .{ }^{12}$ The magnitudes of the estimates for the pure altruism and error parameters seem plausible: the significant pure altruism effect is not surprising given that average contributions rise from 6 to 23 to 58 percent as the group size is increased from 4 to 10 to 40 (when $m=.3$ ). This 'numbers effect' cannot be explained by warm-glow altruism, and the empirical model does not pick up evidence of such

\footnotetext{
${ }^{11}$ Specifically, we used the data from the sessions reported in Isaac and Walker (1988) plus the $n=40$ cash treatments in Isaac et al. (1994). Each sequence consisted of 10 rounds; we used data only from the last five rounds in each case, since then we expect behavior to have stabilized. Thus the data from the first paper consisted of six sequences with $(n, m, \omega)=(4,0.75,25)$, six with $(n, m, \omega)=(4,0.3$, $62)$, six with $(n, m, \omega)=(10,0.75,10)$, and six with $(n, m, \omega)=(10,0.3,25)$; a total of 840 observations. The data from the second paper consisted of three sequences with $(n, m, \omega)=(40,0.3,50)$, and one with $(n, m, \omega)=(40,0.03,50)$, for a further 800 observations. In each case we converted the endowment data into dollar equivalents, so that the estimates are in dollar terms, and can thus be compared across experiments.

${ }^{12}$ In the three-parameter model, warm-glow and pure altruism parameters were highly correlated ( $\rho=-0.98)$. The estimated values for $\mu, \alpha$, and $g$ were $0.036(0.003), 0.085(0.009)$, and -0.18 (0.10) respectively.
} 
altruism. Our model, however, does not explain the absence of a numbers effect for $m=0.75$ in this data set; indeed, there is no evidence of pure altruism when the estimation is restricted to this high value of $m$. This anomaly may drive some of the conflicting results in the literature: a pure-altruism effect is more likely to be found in data sets that include low marginal values of the public good and a large variation in the number of participants.

Our results are quite different (in terms of the break-down of altruism) from those of Palfrey and Prisbrey (1997). ${ }^{13}$ These authors fix the group size at four and vary the value of the private good (which is normalized in the treatments we analyze), and find a significant warm-glow effect and no evidence of pure altruism. In their set-up, an individual either contributes or does not, ${ }^{14}$ and a token kept is worth $r_{i}$ to individual $i$ (private knowledge) while it is worth $V$ to everyone (common knowledge) if contributed. Hence $V / r_{i}$ is analogous to $m$ in our context. In the absence of any altruism, a rational individual would therefore contribute if $r_{i}<V$, and keep the token if $r_{i}>V$. Palfrey and Prisbrey (1997) vary $r_{i}$ between 1 and 20 (in unit increments), while $V$ was taken in different sequences to be 3, 6, 10 , or 15 . Thus it is a weakly dominant strategy to give (i.e., $m>1$ ) for roughly two-fifths of the data points.

Palfrey and Prisbrey (1997) find a value of warm-glow to be around 1.6 for experienced subjects, and 2.2 on average. Since they find no evidence of pure altruism, their estimates translate into a dividing line between contributors and non-contributors of $r=V+2.2$. Roughly, this suggests an absolute 'rule-of-thumb' whereby individuals contribute if their private loss is less than 2.2 units. It is instructive to compare this situation to our own estimates applied to their framework. Since we find a value for $\alpha$ of 0.067 and no warm-glow, when there are 4 players the total pure altruism component, $(n-1) \alpha$, is around 0.2 , so the dividing line between contributing and not would be $r=(1.2) V$. Note that this corresponds loosely to a relative rule-of-thumb ("contribute if the private loss is less than 20\%'). Given the finite grid of possible data points, there are very few data points that fall to one side of the absolute value criterion but fall on the other side of the relative value criterion. Indeed, for the values given, the only points that are thus contentious are $(r, V)=(4,3),(r, V)=(5,3)$, and $(r, V)=(8,6)$. The

\footnotetext{
${ }^{13}$ We find a greater propensity to make errors, presumably because of the greater complexity from choosing a level of contributions rather than facing a simple binary choice in Palfrey and Prisbrey (1997).

${ }^{14}$ In half the treatments, individuals had a single token to give or keep, in the other half, individuals had nine tokens each. It is interesting that in the latter treatments there was little 'splitting' of tokens, especially among experienced subjects, meaning that most players either contributed all their tokens or none at all. This behavior is very different from evidence in the standard game, where there is a preponderance of splitting - relatively few individuals are in corner solutions. One treatment difference is that valuations are private knowledge in Palfrey and Prisbrey (1997), but they are common knowledge (and the same for all players) in the usual set-up. When valuations are private information it is possible that individuals are less inclined to 'signal' to others to increase their contributions.
} 
latter two points are very close to the absolute-value cut-point, so consider the first, At this point, in the Palfrey-Prisbrey experiments, the difference to an individual between contributing and not is 1 , and each such point translated into 15 experimental francs, which, at 100 francs to 11 cents, means a private difference of 1.65 cents. At this level of discrimination, one would expect small differences in circumstances to take over from monetary payoffs. Thus we are lead to conclude that the difference between our results and those of Palfrey-Prisbrey are more illusory than real. Moreover, the actual amounts of altruistic behavior also seem small in absolute value.

The linear model is easily generalized to allow for individual differences in both types of altruism and error parameters. Since the effects of others' contributions enter additively as constants in the expected payoff expressions, these terms factor out of the exponential expressions in the numerator and denominator of Eq. (1). This property, which is unaffected by individual differences in $g, \alpha$, and $\mu$, allows the analysis of asymmetries to parallel that of the symmetric case. Individualspecific $g_{i}, \alpha_{i}$, and $\mu_{i}$ imply that player $i$ 's choices follow an exponential density, given by Eq. (4) with parameter $\lambda_{i}^{*} \equiv\left(1-g_{i}-m-m[n-1] \alpha_{i}\right) / \mu_{i}$. Hence, the equilibrium is asymmetric (meaning that different players have different equilibrium densities), but each player's contributions satisfy the comparative statics properties of Proposition 2. Moreover, the 'sandwich' property mentioned above still holds at the level of the individual player in this asymmetric case. When $\lambda_{i}^{*}>0$, player $i$ 's contribution is zero at the Nash equilibrium (free-riding). In the logit equilibrium, player $i$ 's contribution density declines away from zero, and so the expected contribution lies between zero (the Nash level) and half the endowment. Conversely, when $\lambda_{i}^{*}<0$ and the Nash equilibrium contribution is at the full endowment, the logit density for these 'strong contributors' is increasing and the expected contribution lies between $\omega / 2$ and $\omega$.

\section{The quadratic public goods game}

One particular feature of the linear public goods game presented above is that the Nash equilibrium is a corner solution, so that any errors necessarily lie to one side of the Nash equilibrium. Furthermore, the additive payoff structure leads to dominant strategies in the game without errors and to a related property in the model with errors, i.e. the probability density of contributions is independent of the expected actions of other players. In the quadratic public goods game, the Nash outcome is typically interior, and the equilibrium strategies depend on what players believe others will do, i.e. on the expected contributions. The extension to allow for errors also yields a contribution density that depends on the expected contributions of the other players. In this case, the equilibrium consistency condition is that the expected contribution (used to calculate expected payoffs) is 
equal to the mean of the actual distribution of contributions (determined by the logit probabilistic choice function).

The payoff to an individual is now assumed to be quadratic in the total contribution, $X$ :

$$
\pi_{i}\left(x_{i}\right)=\omega-x_{j}+m X-c X^{2},
$$

where $c>0$, so we have simply subtracted a squared term from the linear payoff. ${ }^{15}$ This formulation corresponds to diminishing marginal value from the public good. We first derive the Nash equilibrium when there is no decision error. When this is interior, it can be found by setting the derivative of Eq. (7) with respect to $x_{i}$ to zero, so $-1+m-2 c X=0$ for all $i$. It is clear that there is a continuum of Nash equilibria, at any one of which the total contribution satisfies

$$
X^{*}=\frac{m-1}{2 c},
$$

as long as this lies between 0 and $n \omega$, which requires that $1<m<2 c n \omega+1$. If the sum of others' contributions is less than this amount, the best response for a player is to 'top up' the total contribution. ${ }^{16}$

Unlike the linear case, the total Nash equilibrium contribution now depends on the parameters in a continuous fashion, increasing in $m$ and decreasing in $c$. Since $n$ does not appear on the right side of Eq. (8), total contributions are independent of the number of players at the Nash equilibrium without pure altruism. ${ }^{17}$ This independence property is the basis of an experiment by Laury et al. (1997). They found that doubling the number of subjects (from five to ten) did actually increase total contributions, whereas (mean) individual contributions decreased. ${ }^{18}$

The effect of the number of players on both total and individual contributions is consistent with the theoretical model once we add altruism. The inclusion of an additive altruism term as in Eq. (6), i.e. $\alpha$ times the sum of the others' payoffs, causes the linear and quadratic parameters, $m$ and $c$, to be replaced by $m(1+\alpha(n-$ $1)$ ) and $c(1+\alpha(n-1))$. The total Nash equilibrium contribution becomes

\footnotetext{
${ }^{15}$ Although $m$ is no longer the marginal private benefit of contribution, a higher $m$ still implies a higher marginal valuation for any contribution level.

${ }^{16}$ One could modify the model slightly by assuming that the value of the private good is non-linear in order to tie down a unique and symmetric outcome. We begin with the form Eq. (7) since that is the design used in several experiments (Isaac and Walker, 1996; Laury et al., 1997, for example). Non-linear private values are considered in Section 3.2 below. Finally, note that the limit of the logit equilibrium as $\mu$ tends to zero picks out the symmetric equilibrium.

${ }^{17}$ With warm-glow alone, a term $g$ is added to the numerator in Eq. (8), and total contributions are still independent of the number of players. For clarity we shall set $g$ to zero in the analysis that follows.

${ }^{18}$ Moreover, the levels of individual contributions were well above the Nash predictions. This finding is consistent with altruism, but it is also consistent with the error model (even without altruism). Indeed, as we show below, in the error model the average contribution exceeds the Nash level when the latter is low relative to the endowment, as was the case in the experiments of Laury et al. (1997).
} 


$$
X^{*}(\alpha)=\frac{m(1+\alpha(n-1))-1}{2 c(1+\alpha(n-1))},
$$

which reduces to Eq. (8) when $\alpha=0$. It follows from Eq. (9) that the total equilibrium contribution, $X^{*}(\alpha)$, is increasing in $n$ if there is altruism $(\alpha>0)$, which provides an explanation for any tendency of contributions to exceed the Nash prediction in Eq. (8). However, altruism alone cannot explain the experimental finding of Isaac and Walker (1996) that total contributions are systematically below the Nash prediction when the latter is high relative to the endowment. These authors also show that total contributions are above the Nash prediction when the Nash outcome is relatively small, and that the Nash equilibrium outcome provides a good prediction of the mean level when it is close to $\omega / 2$ per capita.

These latter findings suggest that errors could play an important role in any explanation of contributions, as is borne out in the analysis below. Moreover, altruism is not the only explanation for the group-size effect in the Laury et al. (1997) experiment. As we show below, the error model also predicts that total contributions rise with the number of players, while individual contributions fall.

\subsection{The model with errors}

We now turn to the derivation of the equilibrium contribution density when players make decision errors. For simplicity of presentation, we shall begin with the case of no altruism. The first step is to modify the quadratic payoff function in Eq. (7) to specify player $i$ 's payoff when other players' actions are stochastic. To do this, write $X^{2}$ as $\left(x_{i}+\Sigma_{j \neq i} x_{j}\right)^{2}$. Expanding this expression, taking expectations, and substituting into Eq. (7), one obtains:

$$
\pi_{i}^{\mathrm{e}}\left(x_{i}\right)=\omega-x_{i}+m x_{i}+m \sum_{j \neq i} E_{i}\left(x_{j}\right)-c x_{i}^{2}-2 c x_{i} \sum_{j \neq i} E_{i}\left(x_{j}\right)-c E_{i}\left\{\sum_{j \neq i} x_{j}\right\}^{2} .
$$

As before, additive constants in the expected payoff cancel out of the exponential expressions of the numerator and denominator of Eq. (1), and we can therefore discard all terms that are independent of $x_{i}$ from Eq. (10). The density of $i$ 's actions, given the densities of other players' actions, follows from Eq. (1):

$$
f_{i}\left(x_{i}\right)=K_{i 1} \exp \left\{\left(-x_{i}+m x_{i}-c x_{i}^{2}-2 c x_{i} \sum_{j \neq i} E_{i}\left(x_{j}\right)\right) / \mu\right\},
$$

where the constant $K_{i 1}$ ensures that the density integrates to unity over $[0, \omega]$. Completing the square in the exponential in Eq. (11) and putting terms that are independent of $x_{i}$ into the constant yields a truncated normal distribution:

$$
f_{i}\left(x_{i}\right)=K_{i 2} \exp \left\{-\frac{c}{\mu}\left[x_{i}-X^{*}+\sum_{j \neq i} E_{i}\left(x_{j}\right)\right]^{2}\right\}, x_{i} \in[0, \omega],
$$


where $K_{i 2}$ is a constant of integration and $X^{*}$ is the total Nash contribution given by Eq. (8). ${ }^{19}$ This is not an explicit solution for the equilibrium density, since the expectation terms on the right-hand side depend on the densities used by other players.

The equilibrium consistency condition is that the expected contributions used to calculate expected payoffs are equal to the corresponding means of the actual equilibrium distributions, i.e. beliefs should be correct on average. The equations that determine expected contributions are given by:

$$
E_{i}\left(x_{j}\right)=\int_{0}^{\omega} x f_{j}(x) \mathrm{d} x, i \neq j .
$$

This is a consistency condition because the densities $f_{j}(x)$ are themselves functions of the expected contributions. Although there is no closed-form solution to Eq. (13), the existence of a solution is guaranteed by the following fixed-point argument. Any vector of expected contributions determines a vector of densities from Eq. (12), which in turn determines a vector of expected contributions, as per Eq. (13). Since each expected contribution lies between 0 and $\omega$, this process defines a continuous mapping from a compact set into itself. Brouwer's theorem then ensures the existence of a solution for the expected contributions. In turn, such a solution completely characterizes the logit equilibrium densities by Eq. (12). In Appendix A, we furthermore show that the equilibrium is unique and symmetric across players.

Proposition 3. For the quadratic public goods game, there exists a unique logit equilibrium, which is symmetric across players. The equilibrium density is a (truncated) normal density.

The normal distribution results from the composition of the quadratic payoff and the exponential logit function. Its precise form is given by Eq. (12), with all the player-specific subscripts suppressed (since the density is symmetric across players), and all the $E^{\prime}$ 's replaced by $E^{*}$, which is the unique solution to Eq. (13). The uniqueness of the logit equilibrium is interesting in light of the continuum of Nash equilibria in this quadratic game. Since the equilibrium is symmetric for any value of the error parameter, $\mu$, the limit distribution has all its mass at the symmetric Nash equilibrium when $\mu$ goes to zero, and in this limit each player therefore contributes $x^{*}=X^{*} / n^{20}$

The analogue of the sandwich result of the previous section is given in the next proposition, which is proved in Appendix A.

\footnotetext{
${ }^{19}$ It is readily checked that expanding the term in the exponential in Eq. (12) gives the term in the exponential in Eq. (11), plus additional terms that are independent of $x_{i}$ : the latter are accounted for in $K_{i 2}$.

${ }^{20}$ Alternatively, $x^{*}$ is the average of the Nash contributions at any asymmetric Nash equilibrium.
} 
Proposition 4. In the logit equilibrium of the quadratic public goods game, the expected contribution per person is sandwiched between the symmetric Nash equilibrium level, $x^{*}$, and half the endowment, $\omega / 2$.

This condition implies that the mean contribution equals the symmetric Nash contribution when the latter is located at $x^{*}=\omega / 2$. The sandwich condition is consistent with the experimental findings in Isaac et al. (1994); Laury et al. (1997). In particular, Isaac et al. (1994) report mean contribution levels that are roughly centered around the symmetric Nash prediction when the Nash equilibrium leaves equal room for error above and below, as shown in Fig. 1. ${ }^{21}$ The upper-left panel shows data for periods 1-10 for the linear public goods game $(c=0)$, so the Nash equilibrium is at zero. The solid line shows the mean contribution, period by period, and the dotted lines indicate a $90 \%$ confidence interval. In this treatment, mean contributions decline from just below half of the endowment (31) towards zero. In the lower-left panel, the Nash equilibrium is at the midpoint of the endowment, and the data are approximately centered around that level. In the two panels on the right side of the figure, the data are sandwiched between the midpoint of the endowment and the Nash equilibrium, which is either 19 tokens above or below $\omega / 2$.

The comparative static properties of the equilibrium distribution for the quadratic game are qualitatively similar to those of the linear game, except for the following one, which is proved in Appendix A. ${ }^{22}$

Proposition 5. In the logit equilibrium of the quadratic public goods game (in the absence of altruism), an increase in the number of players leads to a stochastic decrease in individual contributions, but to a stochastic increase in total contributions.

This property is confirmed by experimental evidence in Laury et al. (1997), who conducted a quadratic public goods experiment with $\omega=125$, and $X^{*}=100$. When there were 5 players per group, the mean contribution was 43 , which is between $x^{*}=20$ and $\omega / 2=62.5$, as predicted by the sandwich property. When the number of players was doubled to 10 , mean contributions dropped to 31 . However, total contributions rose (from 217 to 306), in line with Proposition 5. These comparative statics results are summarized on the right side of Table 1. As for the linear game, the logit model provides better qualitative predictions than Nash.

For completeness, we now describe the equilibrium density in the presence of both altruism and errors. As we noted at the beginning of this subsection, setting $\alpha=0$ is merely an algebraic convenience. For $\alpha>0$, it is straightforward to show

\footnotetext{
${ }^{21}$ This figure is similar to figure 3 in Isaac and Walker (1996).

${ }^{22}$ That is, contributions stochastically increase with $m$ and $\omega$, while they stochastically increase (decrease) with $\mu$ when $x^{*}<\omega / 2\left(x^{*}>\omega / 2\right)$. The proofs are similar to that of Proposition 5 .
} 

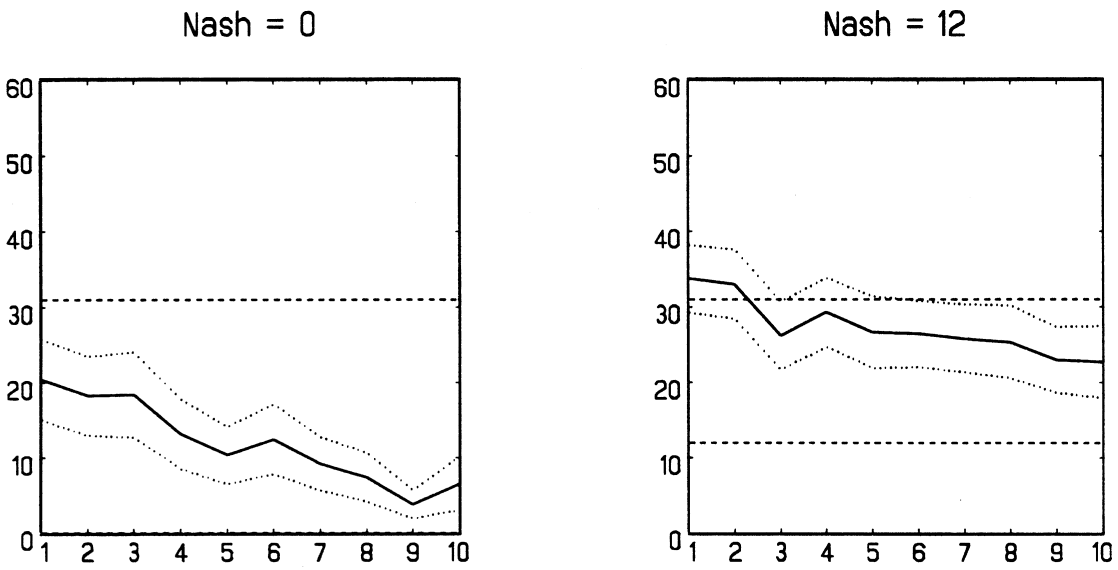

Nash $=31$

Nash $=50$
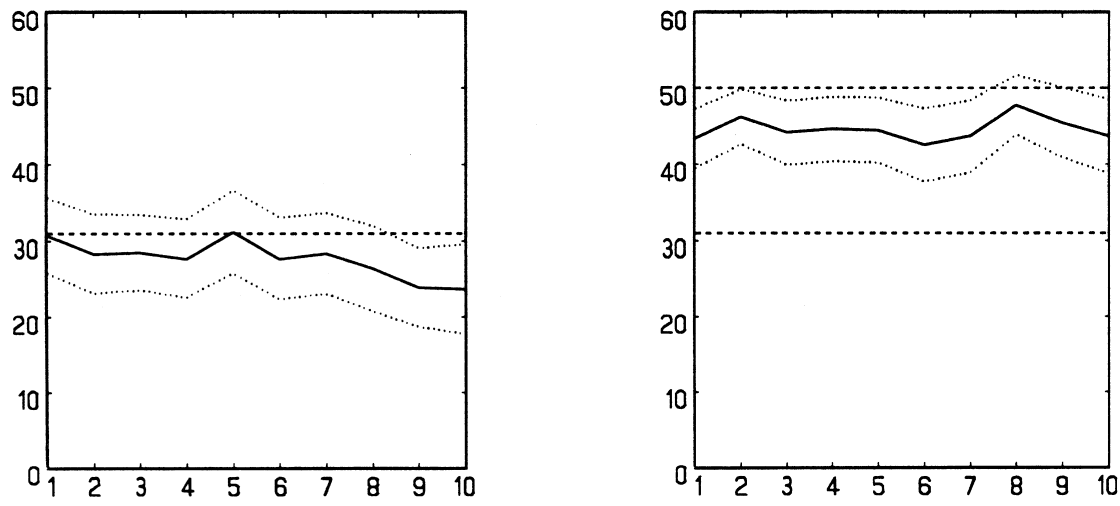

Fig. 1. Source: Isaac and Walker (1994).

that the probability density Eq. (12) is modified by simply replacing $c$ by $c(1+\alpha(n-1))$ and $X^{*}$ by the altruism-inclusive form, Eq. (9). Intuitively, these correction factors follow from the fact that there are $(n-1)$ other players, each of whose payoffs is valued per dollar at $\$ \alpha$. Since these changes simply amount to rescaling constants, the analysis from Appendix A follows through directly, so there is a unique equilibrium, and again the sandwich property holds: the mean contribution lies between half the endowment and the (altruism-inclusive) Nash equilibrium.

The quadratic model can easily be generalized to allow for individual differ- 
ences in error parameters. As for the symmetric case, we can prove that there exists a unique logit equilibrium, which is now asymmetric across players. Each player's density is given by a truncated normal, and in parallel to Proposition 4, each player's expected contribution is sandwiched between that player's Nash contribution and half the endowment. ${ }^{23}$ Nevertheless, it is important to recognize that even the symmetric case generates dispersion in the actual play since each player's observed action is a draw from the equilibrium density.

\subsection{An alternative quadratic specification}

Keser (1996) considers a variant of the quadratic game in which there is diminishing marginal value of the private good, rather than a diminishing marginal value of the public good. Instead of Eq. (7) she assumes

$$
\pi_{i}\left(x_{i}\right)=\omega-x_{i}-\gamma\left(\omega-x_{i}\right)^{2}+m X,
$$

where $\gamma>0$. Since Eq. (14) is also a quadratic function of a player's own contribution, the logit exponential formula again leads to a truncated normal distribution in the model with errors. Another interesting feature of this formulation is that, as in the linear public goods game, the Nash equilibrium is a dominant strategy, since the derivative of Eq. (14) with respect to $x_{i}$ is independent of others' contributions. The Nash contribution per player is

$$
x^{*}=\frac{m-1}{2 \gamma}+\omega
$$

as long as this lies between 0 and $\omega$. In contrast with the linear model of Section 2, this is an interior, dominant-strategy equilibrium.

When players make decision errors (and for $\alpha=0$ ) we can follow our development of equations Eqs. (11) and (12) to first derive the probability density of $i$ 's actions as

$$
f_{i}\left(x_{i}\right)=K_{3} \exp \left\{\left((m+2 \gamma \omega-1) x_{i}-\gamma x_{i}^{2}\right) / \mu\right\}
$$

with $K_{3}$ ensuring the density integrates to one over its support, $[0, \omega]$. We again complete the square in the exponential, put terms that are independent of $x_{i}$ into the constant, and use Eq. (15) to give a truncated normal distribution:

$$
f_{i}\left(x_{i}\right)=K_{4} \exp \left\{-\frac{\gamma}{\mu}\left(x_{i}-x^{*}\right)^{2}\right\}, x_{i} \in[0, \omega],
$$

where $K_{4}$ is a constant of integration and the Nash contribution, $x^{*}$, is now given

\footnotetext{
${ }^{23}$ More precisely, if the players are labeled so that $\mu_{1} \leq \mu_{2} \leq . . \leq \mu_{n}$, the expected contributions, $E_{i}$, are ranked $x^{*} \leq E_{1} \leq E_{2} \leq . . \leq E_{n} \leq \omega / 2$ when the Nash contribution is below half the endowment, and $x^{*} \geq E_{1} \geq E_{2} \geq . . \geq E_{n} \geq \omega / 2$, when it is above half the endowment. In other words, the lower one's $\mu$, the closer the expected contribution is to the Nash contribution.
} 
by Eq. (15). In comparison to Eq. (12), there is no consistency condition to verify, essentially because the Nash equilibrium involves playing a dominant strategy.

The density in Eq. (17) is a truncated normal with the mode at the Nash equilibrium, and so the analogous result to that of Section 3.1 holds: the mean contribution is sandwiched between the Nash equilibrium and half the endowment. (Were we to add altruism, the mean contribution would move upward.) Keser's experiment sets $\omega=20$, and the Nash equilibrium at $x^{*}=7$. Over a sequence of 25 trials, she reports that the mean contribution consistently exceeded 7 . In the last five periods, the mean contribution was 8.9, again well in line with our sandwich proposition. Keser notes: 'If we suppose that errors are equally likely in both directions then, on average, they should cancel out. Thus the observed overcontribution in our experiment cannot be interpreted as resulting from subjects' error-making' (Keser, 1996, p. 3). As we have shown, endogenous errors do not cancel out, but rather lead to a systematic bias as compared to the Nash equilibrium. This underscores the importance of formal modeling of errors.

\section{Conclusion}

Laboratory experiments often yield 'noisy' results in the sense that choices are scattered rather than identical across subjects. Furthermore, systematic bias is often observed insofar as mean choices lie above or below the equilibrium predicted from the theoretical model underlying the experiment. There are several ways to explain these observations. First, monetary payoffs may not exactly reflect the true payoffs that motivate subjects' actions. For example, subjects may care about how well they do relative to others (envy, fairness), or about how well others do (altruism), or they may be risk averse. Once we admit the possibility that unobservable factors can influence intrinsic payoffs, the scatter in the data might be explained by individual heterogeneity in preferences. Another source of scatter may be that subjects make errors in evaluating their best decisions. This can happen through confusion as to the way payoffs are determined, miscalculation of actual returns, or misjudgment of what other players will do.

It seems intuitively likely that several of the factors listed above will be at work in any given context, with their relative importance depending on the particular experiment. In this paper, we concentrate on two factors, errors and altruism, which are commonly mentioned as explanations for 'excess' contributions in linear public goods games with boundary, zero-contribution Nash equilibria. To get precise results, we have assumed that these effects enter in a specific manner. As in some previous work, altruism is postulated to enter utilities additively. Our main contribution is the theoretical treatment of errors. The model supposes that a small error (in terms of a subject choosing an action yielding a lower payoff than the maximum possible) is more likely than a large one, thus reflecting a form of bounded rationality. Following McKelvey and Palfrey (1995), the model is one of 
equilibrium errors in that mistakes made by others are accounted for when we determine the equilibrium density of actions (here the contributions to a public good). In particular, the distributions of others' decisions determine each player's expected payoffs, and these expected payoffs in turn determine the probability density of each player's contribution decision. The equilibrium is a fixed point of this process.

One criticism of the logit equilibrium approach has been that it seems too complicated for boundedly rational individuals to figure out. It would indeed be contradictory to the spirit of bounded rationality that agents could calculate the expected error-prone actions of others accurately, and then make errors in a comparatively simple decision problem. However, just as the pool-player in the famous analogy can shoot well without calculating the coefficient of restitution between the ball and the cushion, in our version of this analogy the pool-player makes mistakes, but not systematic ones. The evolutionary process described in Anderson et al. (1997) provides a related response: when people only change their decisions in the direction of increasing payoffs, subject to random noise, behavior converges to a logit equilibrium. This is analogous to the way that a Nash equilibrium might be reached as individuals make best responses to observed frequencies of past decisions. It is the noise in the local adjustment process in our model that causes the steady state to differ from Nash, but we are not assuming more rationality than in a standard model of learning that leads to a Nash equilibrium.

Let us briefly recap some of the results of this paper. We prove existence, uniqueness, and symmetry of the logit equilibrium, for both linear and quadratic public goods games. The comparative statics predictions of the model are roughly consistent with the stylized facts about behavior in laboratory experiments in that: (i) contributions are interior, and the mean contribution is sandwiched between the Nash equilibrium and half the endowment, (ii) contributions (stochastically) increase with the marginal value of the public good and with the endowment, and (iii) total contributions are increasing in the number of participants in the presence of altruism. Also, the evolutionary adjustment process mentioned above can be used to generate contribution patterns that decline over time, as observed in experiments. The model, however, does not allow for strategic interactions across periods, and therefore, it cannot explain patterns in contributions that appear to be related to dynamic considerations, e.g. signaling or other attempts to establish and encourage tacit collusion. These strategic stories are important since they can explain why contributions tend to be sensitive to the number of periods remaining in experiments with fixed groupings.

Our results provide some new insights on the effects of altruism and decision error. For the linear public good game in which positive contributions are often observed, the Nash equilibrium entails zero contributions. Positive contributions may be due to altruism, but altruism alone cannot explain why most subjects contribute less than the total endowment when the Nash equilibrium is at full 
contributions. $^{24}$ These observations are explained by our error model, since it predicts that subjects err away from the Nash predictions. However, a positive taste for altruism is needed in addition to the error hypothesis to explain the experimental finding that a greater number of players increases the total contribution. The quadratic public goods game also underscores the importance of the error model in explaining experimental results. When the symmetric Nash equilibrium lies at half the endowment, $\omega / 2$, experimental evidence is that roughly half of the subjects over-contribute reltive to this benchmark, and the others under-contribute. The scatter around the Nash prediction when it is in the middle of the range of possible contributions is predicted by our error model. More generally, the model predicts mean contributions to lie between $\omega / 2$ and the Nash equilibrium, which is roughly consistent with the 'sandwich pattern' observed in experimental results.

The logit model produces specific functional forms that can be useful for estimation: the equilibrium is a truncated exponential in the linear public goods game, and it is a truncated normal for the quadratic game. The closed-form solution for the linear public goods game makes maximum likelihood estimation straightforward (unlike the quadratic case where the functional form is known but there is no closed-form solution). We used data from previous linear public goods experiments to estimate pure altruism and error parameters; both were significant and of reasonable magnitude, but warm-glow was insignificant. Although the estimation included data from sessions with both low and high marginal values of the public good, our model does not explain the apparent absence of a numbers effect for treatments with a high $m$.

Subsequent modifications of the basic theory may prove useful in explaining other features in the data. For example, behavioral heterogeneity across individuals suggests models with individual differences in altruism and the propensity to make errors. In any case, the value of having a formal theory (the one presented here, or some modification of it) is that it is useful in organizing the data, in suggesting future experiments, and in the specification of econometric tests.

\section{Acknowledgements}

We wish to thank Maxim Engers, Bill Johnson, Claudia Keser, Susan Laury, Ed Olsen, Jeff Prisbrey, Roger Sherman, Steve Stern, and two anonymous referees for helpful comments, and Mark Isaac and James Walker for supplying the laboratory

\footnotetext{
${ }^{24}$ These results could also be explained by some subjects being altruistic and others envious. A given subject could also be altruistic when others receive little and envious when others receive a lot. Yet such an explanation strains the imagination somewhat because the evidence for the quadratic public goods game calls for the altruistic subjects to roughly offset the envious ones when the Nash equilibrium is at half of the endowment.
} 
data. This research was funded in part by the National Science Foundation (SBR-9617784).

\section{Appendix A}

Lemma 1. The logit equilibrium distribution function in Eq. (5) is strictly increasing in $\lambda$ for $0<x<\omega$.

Proof. The derivative of the right-hand side of Eq. (5) with respect to $\lambda$ is strictly positive if and only if $x \exp (-\lambda x)(1-\exp (-\lambda \omega))>\omega \exp (-\lambda \omega)(1-\exp (-\lambda x))$. Multiplying through by $\exp (\lambda(x+\omega))$ and rearranging yields:

$$
\frac{\exp (\lambda \omega)-1}{\omega}>\frac{\exp (\lambda x)-1}{x}
$$

for all positive $x$. Since the two sides are equal at $x=\omega$, it suffices that the right side of this inequality increase in $x$, which holds if $1-\exp (-\lambda x)-\lambda x<0$. This last inequality holds for all interior $x$, since the left side is strictly concave in $x$, with a maximum of 0 at $x=0$. Q.E.D.

Proof of Proposition 3. We first show (i) that any equilibrium is necessarily symmetric, and then (ii) that any symmetric equilibrium is unique. To simplify the presentation we set $c / \mu=1$.

To prove (i), first note that the consistency condition Eq. (13) implies that each player's expectation of player $j$ 's contribution is the same. Let $E_{j}$ denote the common expected contribution of player $j$, i.e. $E_{j}=E_{i}\left(x_{j}\right)$ for all $i$. Rewrite Eq. (13) as:

$$
\int_{0}^{\omega}\left(x-E_{j}\right) f_{j}(x) \mathrm{d} x=0, j=1, \ldots, n,
$$

which follows since the density $f_{j}(x)$ integrates to one. Substituting for $f_{j}(x)$ from Eq. (12), and dividing both sides by the constant $K_{j 2}$, yields:

$$
\int_{0}^{\omega}\left(x-E_{j}\right) \exp \left(-\left(x-X^{*}+\sum_{i \neq j} E_{i}\right)^{2}\right) \mathrm{d} x=0, j=1, \ldots, n .
$$

Let $\Omega=\Sigma_{j} E_{j}$ denote total expected contributions over all $n$ players. We can rewrite Eq. (A.1) as

$$
\int_{-E_{j}}^{\omega-E_{j}} y \exp \left(-\left(y-X^{*}+\Omega\right)^{2}\right) \mathrm{d} y=0, j=1, \ldots, n,
$$

where we have shifted the integration variable from $x$ to $y=x-E_{j}$. Notice that the integrand is player independent. Since it is strictly negative for $y<0$, and strictly 
positive for $y>0$, these conditions can only be satisfied if the expected contribution $E_{j}$ is the same for all players. Hence, any logit equilibrium is symmetric.

Next, we prove (ii). To this end, define $z(x ; n, E)=x+(n-1) E-X^{*}$, where $E$ denotes the expected contribution of each player. Under symmetry, the equilibrium consistency condition Eq. (A.1) becomes:

$$
\int_{0}^{\omega}(x-E) \exp \left(-z(x ; n, E)^{2}\right) \mathrm{d} x \equiv H(n, E)=0
$$

A solution, $E^{*}$, to Eq. (A.3) uniquely ties down the equilibrium density, and so it remains to show that there is only one solution to Eq. (A.3). We prove this by showing that the derivative of $H$ is negative at any solution, $E^{*}$ :

$$
\begin{aligned}
& \frac{\partial H\left(n, E^{*}\right)}{\partial E}=-\int_{0}^{\omega} \exp \left(-z\left(x ; n, E^{*}\right)^{2}\right) \mathrm{d} x \\
& -2(n-1) \int_{0}^{\omega}\left(x-E^{*}\right)\left[\left(x-E^{*}\right)+n E^{*}-X^{*}\right] \exp \left(-z\left(x ; n, E^{*}\right)\right)^{2} \mathrm{~d} x .
\end{aligned}
$$

The first term is negative, and, since $n E^{*}-X^{*}$ is independent of $x$, Eq. (A.3) implies that the second term is equal to:

$$
-2(n-1) \int_{0}^{\omega}\left(x-E^{*}\right) \exp \left(-z\left(x ; n, E^{*}\right)^{2}\right) \mathrm{d} x .
$$

Hence $\partial H\left(n, E^{*}\right) / \partial E<0$. Since $H$ is continuous in $E$ and strictly decreasing in $E$ at any possible solution, Eq. (A.3) has a unique solution. Q.E.D.

Proof of Proposition 4. Recall that the equilibrium density is proportional to $\exp \left(-z(x ; n, E)^{2}\right)$, where $z(x ; n, E)=x+(n-1) E-X^{*}$. Consider the case $x^{*}=\omega / 2$. We show that $E=\omega / 2$ is a solution to Eq. (A.3), and, by Proposition 3, this is the unique solution. Indeed, since $z(x ; n, \omega / 2)=x-\omega / 2$ when $x^{*}=\omega / 2$, the exponential term in Eq. (A.3) is symmetric around $\omega / 2$, and so Eq. (A.3) holds. Now consider the case $x^{*}>\omega / 2$, and let the mode of the equilibrium density be denoted by $T$, so that $T=n x^{*}-(n-1) E^{*}$ from the definition of $z$. Clearly, $E^{*}>x^{*}>\omega / 2$ is not a solution since then $T<x^{*}$ and $H$ would be negative since most of the probability density would lie below $E^{*}$ (see Eq. (A.3)). Hence mean contributions, $E^{*}$, must lie below the symmetric Nash level, $x^{*}$. However, the mean contribution still exceeds $\omega / 2$ : for $E^{*}<x^{*}$ we have $T>x^{*}$, but if $E^{*}$ were less than $\omega / 2$ then most of the probability density would be above $E^{*}<\omega / 2$, so $H$ would be positive, a contradiction. Similar results for $x^{*}<\omega / 2$ establish that mean contributions then lie above the Nash level, but below $\omega / 2$. Q.E.D.

Proof of Proposition 5. Applying the implicit function theorem to $H\left(n, E^{*}\right)=0$, yields the comparative static effect of the number of players, $n$, on the equilibrium expected contribution, $E^{*}$, as: 


$$
\frac{\mathrm{d} E^{*}}{\mathrm{~d} n}=-\frac{\partial H\left(n, E^{*}\right) / \partial n}{\partial H\left(n, E^{*}\right) / \partial E} .
$$

The numerator of the right side is given by:

$$
\frac{\partial H}{\partial n}=-2 E^{*} \int_{0}^{\omega}\left(x-E^{*}\right)\left[\left(x-E^{*}\right)+n E^{*}-X^{*}\right] \exp \left(-z(x ; n, E)^{2}\right) \mathrm{d} x,
$$

which is strictly negative because both the expected contribution, $E^{*}$, and the integral are positive, see the proof of Proposition 3. Since $\partial H\left(n, E^{*}\right) / \partial E<0$ (also from the proof of Proposition 3), then $\mathrm{d} E^{*} / \mathrm{d} n<0$, i.e. the (mean) individual contribution falls with the number of players.

To show that total contribution, $\Omega$, rises with the number of players, write the equilibrium consistency condition Eq. (A.2) under symmetry as:

$$
\int_{-E^{*}}^{\omega-E^{*}} y \exp \left(-\left(y-X^{*}+\Omega\right)^{2}\right) \mathrm{d} y=0 .
$$

Since we know that $E^{*}$ decreases with the number of players, $n$, the region of integration shifts to the right as $n$ increases. To maintain the above equality, the mode of the exponential term has to shift to the left. The mode is at $X^{*}-\Omega$, and since $X^{*}$ is independent of $n$, the total expected contribution $\Omega$ has to increase with $n$. Q.E.D.

\section{References}

Anderson, S.P., de Palma, A., Thisse, J.-F., 1992. Discrete Choice Theory of Product Differentiation. MIT Press, Cambridge MA.

Anderson, S.P., Goeree, J.K., Holt, C.A., 1997. Stochastic game theory: adjustment to equilibrium under bounded rationality. University of Virginia working paper 304.

Andreoni, J., 1988. Why free ride? Strategies and learning in public goods experiments. Journal of Public Economics 37, 291-304.

Andreoni, J., 1993. An experimental test of the public goods crowding-out hypothesis. American Economic Review 83, 1317-1327.

Andreoni, J., 1996. Cooperation in public goods experiments: kindness or confusion? American Economic Review 85, 891-904.

Andreoni, J., Miller, J.H., 1995. Giving according to GARP: an experimental study of rationality and altruism. University of Wisconsin-Madison working paper.

Brandts, J., Schram, A., 1996. Cooperative gains or noise in public good experiments: applying the contribution function approach. Tinbergen Institute Discussion Paper 96-81/1, University of Amsterdam.

Chen, H.-C., Friedman, J.W., Thisse, J.-F., 1997. Boundedly rational Nash equilibrium: a probabilistic choice approach. Games and Economic Behavior 18, 32-54.

Croson, R., 1996. Partners and strangers revisited. Economics Letters 53, 25-32.

Davis, D.D., Holt, C.A., 1993. Experimental Economics. Princeton University Press, Princeton NJ.

DeGroot, M.H., Cyert, R.M., 1973. An analysis of cooperation and learning in a duopoly context. American Economic Review 63, 24-37. 
Edgeworth, F.Y., 1881. Mathematical Psychics. Kegan Paul, London UK.

Holt, C.A., Laury, S.K., 1997. Theoretical explanations of treatment effects in voluntary contributions experiments. In: Plott, C., Smith, V. (Eds.), Handbook of Experimental Economics Results. Elsevier, New York NY, forthcoming.

Isaac, M., Walker, J.M., 1988. Group size effects in public goods provision; the voluntary contributions mechanism. Quarterly Journal of Economics 103, 179-199.

Isaac, M., Walker, J.M., 1996. Nash as an organizing principle in the voluntary provision of public goods: experimental evidence. University of Arizona working paper.

Isaac, M., Walker, J.M., Williams, A.W., 1994. Group size and the voluntary provision of public goods: experimental evidence utilizing large groups. Journal of Public Economics 54, 1-36.

Keser, C., 1996. Voluntary contributions to a public good when partial contribution is a dominant strategy. Economics Letters 50, 359-366.

Kelley, H.H., Stahelski, A.J., 1970. Errors in perception of intentions in a mixed-motive game. Journal of Experimental Psychology 6, 379-400.

Kreps, D., Milgrom, P., Roberts, J., Wilson, R., 1982. Rational cooperation in the finitely repeated prisoner's dilemma. Journal of Economic Theory 27, 245-252.

Laury, S.K., 1997. Alternatives to the Nash model in the voluntary contribution mechanism environment. University of South Carolina working paper.

Laury, S.K., Walker, J.M., Williams, A.W., 1997. The voluntary contribution mechanism: provision of a pure public good with diminishing marginal returns. Public Choice, forthcoming.

Ledyard, J.O., 1995. Public goods: A survey of experimental research. In: Kagel, J.H., Roth, A.E. (Eds.), The Handbook of Experimental Economics. Princeton University Press, Princeton NJ, pp. 111-194.

Lopez, G., 1995. Quantal response equilibria in posted offer auctions. University of Virginia doctoral dissertation.

Luce, D.R., 1959. Individual choice behavior. John Wiley \& Sons, Ltd. New York, NY.

McKelvey, R.D., Palfrey, T.R., 1995. Quantal response equilibria for normal form games. Games and Economic Behavior 10, 6-38.

Miller, J., Andreoni, J., 1991. Can evolutionary dynamics explain free riding in experiments? Economics Letters 36, 9-15.

Mirrlees, J.A., 1986. Economic policy and nonrational behaviour. Nuffield College, Oxford, working paper.

Ochs, J., 1995. Games with unique mixed-strategy equilibria: an experimental study. Games and Economic Behavior 10, 202-217.

Offerman, T., Sonnemans, J., Schram, A., 1996. Value orientations and voluntary contributions in public goods. Economic Journal 106, 817-845.

Offerman, T., Schram, A., Sonnemans, J., 1996. Quantal response models in step-level public goods games. European Journal of Political Economy, forthcoming.

Olson, M., 1971. The Logic of Collective Action. Harvard University Press, Cambridge MA.

Palfrey, T.R., Prisbrey, J.E., 1997. Anomalous behavior in linear public goods experiments: how much and why? American Economic Review 87, 829-846.

Rosenthal, R.W., 1989. A bounded rationality approach to the study of noncooperative games. International Journal of Game Theory 18, 273-292.

Sefton, M., Steinberg, R., 1996. Reward structures in public goods experiments. Journal of Public Economics 61, 263-287. 